\title{
Misiurewicz maps unfold generically (even if they are critically non-finite)
}

by

\author{
Sebastian van Strien (Warwick)
}

\begin{abstract}
We show that in normalized families of polynomial or rational maps, Misiurewicz maps (critically finite or infinite) unfold generically. For example, if $f_{\lambda_{0}}$ is critically finite with non-degenerate critical point $c_{1}\left(\lambda_{0}\right), \ldots, c_{n}\left(\lambda_{0}\right)$ such that $f_{\lambda_{0}}^{k_{i}}\left(c_{i}\left(\lambda_{0}\right)\right)=p_{i}\left(\lambda_{0}\right)$ are hyperbolic periodic points for $i=1, \ldots, n$, then

$$
\lambda \mapsto\left(f_{\lambda}^{k_{1}}\left(c_{1}(\lambda)\right)-p_{1}(\lambda), \ldots, f_{\lambda}^{k_{d-2}}\left(c_{d-2}(\lambda)\right)-p_{d-2}(\lambda)\right)
$$

is a local diffeomorphism for $\lambda$ near $\lambda_{0}$. For quadratic families this result was proved previously in $[\mathrm{DH}]$ using entirely different methods.

1. Introduction and statement of results. In this paper we consider families of polynomial or rational maps $f_{\lambda}$ depending (complex) analytically on $\lambda \in V$ where $V$ is some neighbourhood of $\lambda_{0} \in \mathbb{C}^{\mathcal{N}}$ where $\mathcal{N} \in \mathbb{N}$. We say that $f_{\lambda}$ is a normalized family of polynomial (resp. rational) maps if $\lambda \neq \lambda^{\prime}$ implies that no affine (resp. Möbius) transformation conjugates $f_{\lambda}$ and $f_{\lambda^{\prime}}$. Let $f=f_{\lambda_{0}}$ and $C(f)$ be the set of critical points of $f$. The map $f$ is called critically finite if each critical point $c_{i} \in C(f)$ of $f$ eventually lands on a periodic point $p_{i}$ of $p$. In other words,

$$
f^{k_{i}}\left(c_{i}\right)=p_{i} \quad \text { and } \quad f^{l_{i}}\left(p_{i}\right)=p_{i}
$$

where for simplicity we choose $k_{i}$ minimal so that $f^{k_{i}}\left(c_{i}\right)$ is a periodic point. We shall only deal with the case where $p_{i}$ are hyperbolic periodic points. In that case $f$ is in the larger class of Misiurewicz maps defined as follows. The map $f$ satisfies the Misiurewicz condition if each critical point $c$ is either in the Julia set or eventually mapped to a periodic point which is hyperbolic (i.e., with multiplier not on the unit circle), and moreover $\omega(c)$ contains no parabolic or critical points. By a theorem of Mañé [Ma2] (see the remarks below our Theorem), these conditions imply that
\end{abstract}

2000 Mathematics Subject Classification: 37F45, 37G99, 37E05, 32H50. 


$$
P(f)=\text { the closure of } \bigcup_{c \in C(f)} \bigcup_{i>0}\left\{f^{i}(c)\right\}
$$

is a hyperbolic set and that all components of the Fatou set of $f$ are in the basins of super-attracting periodic orbits. Note that we only consider forward iterates of critical points in the definition of $P(f)$.

The aim of this paper is to prove the following theorem.

MaIN THEOREM 1.1. Let $f_{\lambda}$ be a normalized family of polynomial or rational maps such that $f=f_{\lambda_{0}}$ is a Misiurewicz map with $n$ critical points $c_{1}, \ldots, c_{n}$. Assume that in the definition above, the critical points $c_{i}$ are all non-degenerate and that $f$ is not a Lattès map (see definition below). Then there exists a neighbourhood $W \subset \mathbb{C}^{\mathcal{N}}$ of $\lambda_{0}$ such that

- $W \ni \lambda \mapsto c_{i}(\lambda)$ is holomorphic;

- there exists a holomorphic motion $\phi: P(f) \times W \rightarrow \overline{\mathbb{C}}$ such that (writing $\left.\phi_{\lambda}(z)=\phi(z, \lambda)\right)$ for $\lambda \in U$ the set $X_{\lambda}=\bigcup_{z \in P(f)}\left\{\phi_{\lambda}(z)\right\}$ is mapped into itself by $f_{\lambda}$ and $f_{\lambda}: X_{\lambda} \rightarrow X_{\lambda}$ is conjugate to $f: P(f) \rightarrow P(f)$ by a homeomorphism $h_{\lambda}$ depending on $\lambda$ with $h_{0}=\mathrm{id}$;

- assuming that $f\left(c_{i}\right)$ are in $\mathbb{C}$, the map

$$
W \ni \lambda \mapsto\left(f_{\lambda}\left(c_{1}(\lambda)\right)-\phi_{\lambda}\left(f\left(c_{1}\right)\right), \ldots, f_{\lambda}\left(c_{n}(\lambda)\right)-\phi_{\lambda}\left(f\left(c_{n}\right)\right)\right)
$$

is an immersion (its derivative has rank $n$ for all $\lambda \in W$ ).

Here we say that $\phi: X \times W \rightarrow \mathbb{C}$ is a holomorphic motion of $X \subset \overline{\mathbb{C}}$ parameterized by $W \ni \lambda_{0}$ with $W$ a subset of some complex manifold if (writing $\phi_{\lambda}=\phi(z, \lambda)$ ) the following properties hold:

1. $\phi_{\lambda_{0}}(z)=z$ for each $z \in X$;

2. $P(f) \ni z \mapsto \phi_{\lambda}(z)$ is one-to-one for each $\lambda \in W$;

3. the map $W \ni \lambda \mapsto \phi(z, \lambda) \in \overline{\mathbb{C}}$ is holomorphic for each $z \in X$.

In the main theorem we do not exclude the possibility that some of the points $f\left(c_{i}\right)$ coincide. If $f$ is critically finite, then the proof shows that the periodic points $p_{i}(\lambda)$ depend holomorphically on $\lambda \in U$ and

$$
\lambda \mapsto\left(f_{\lambda}^{k_{1}}\left(c_{1}(\lambda)\right)-p_{i}(\lambda), \ldots, f_{\lambda}^{k_{n}}\left(c_{n}(\lambda)\right)-p_{n}(\lambda)\right)
$$

is a local immersion.

REMARKs. 1 . We say that $f$ is a Lattès map if the following holds. Let $n>1$ be an integer, $\Lambda$ be a lattice with $n \Lambda \subset \Lambda$ and $T^{2}=\mathbb{C}^{2} / \Lambda$ be a torus. Let $g_{n}: T^{2} \rightarrow T^{2}$ correspond to $z \mapsto n z$. Let $\pi: T^{2} \rightarrow \overline{\mathbb{C}}$ be an even twofold branched covering (for example the Weierstrass function). Since $g_{n}$ is odd, there exists a rational map $f$, called a Lattès map, of degree $n^{2}$ on the sphere so that $\pi \circ g_{n}=f \circ \pi$. Since the periodic points of $g_{n}$ are dense, the Julia set of $f$ is equal to $\overline{\mathbb{C}}$. The critical points of $f$ correspond to critical values of $\pi$ (critical points are branch points of $\pi$ ) and therefore 
$\#(C(f) \cup P(f))=4$. Since there are arcs of such maps (corresponding to $\Lambda$ ) consisting of quasiconformally conjugate maps, the assumption in the main theorem that $f$ is not a Lattès map cannot be dispensed with.

2. The main theorem implies for example that if we normalize and parameterize the space of polynomials of degree $d$ by

$$
f_{\lambda}(z)=z^{d}+a_{d-2} z^{d-2}+\ldots+a_{0}
$$

where $\lambda=\left(a_{d-2}, \ldots, a_{0}\right)$ and consider $f=f_{\lambda_{0}}$ as above, then the map

$$
\lambda \mapsto\left(f_{\lambda}^{k_{1}}\left(c_{1}(\lambda)\right)-p_{1}(\lambda), \ldots, f_{\lambda}^{k_{d-2}}\left(c_{d-2}(\lambda)\right)-p_{d-2}(\lambda)\right)
$$

is a local diffeomorphism for $\lambda$ near $\lambda_{0}$.

3. If $f_{\lambda}$ and $\widetilde{f}_{\lambda}$ are two families which are Möbius equivalent, then the derivatives at $\lambda=\lambda_{0}$ of

$$
\lambda \mapsto\left(f_{\lambda}\left(c_{1}(\lambda)\right)-\phi_{\lambda}\left(f\left(c_{1}\right)\right), \ldots, f_{\lambda}\left(c_{n}(\lambda)\right)-\phi_{\lambda}\left(f\left(c_{n}\right)\right)\right)
$$

and its corresponding object for $\tilde{f}_{\lambda}$ coincide. This is because the right hand side vanishes at $\lambda=\lambda_{0}$ and is the same for $f_{\lambda}$ and $T \circ f_{\lambda}$ for any affine map $T$ and because the derivative with respect to $\lambda$ of (1.1) with $f_{\lambda}$ replaced by $\tilde{f}_{\lambda}=M_{\lambda} \circ f_{\lambda} M_{\lambda}^{-1}$ is the same as with $f_{\lambda}$ replaced by $T \circ f_{\lambda}$ where $T=D M_{\lambda_{0}}(0)$.

4. If $f_{\lambda}$ is a normalized family then $\mathcal{N} \leq n$. Moreover, any normalized family can be (locally) embedded in a normalized family with $\mathcal{N}=n$. This holds because one can consider the space of all polynomial (resp. rational) maps of the degree considered and define $f \sim g$ if $f$ and $g$ are affinely (resp. Möbius) conjugate. Each equivalence class is a two-dimensional (resp. threedimensional) complex manifold. Indeed, if $f(z)=a_{0} z^{n}+a_{1} z^{n-1}+\ldots+a_{n}$ with $a_{0} \neq 0$ and $M(z)=\alpha z+\beta$ then $M^{-1} \circ f \circ M(z)=a_{0} \alpha^{n-1} z^{n}+$ $\alpha^{n-2}\left(a_{0} n \beta+\alpha^{n-2} a_{1}\right) z^{n-1}+\ldots$, which is a two-parameter family in $\alpha, \beta$. If $f$ is rational of degree $n$ we can write

$$
f(z)=\frac{a_{0} z^{n}+\ldots+a_{n}}{b_{0} z^{n}+\ldots+b_{n}}
$$

such that $\left(a_{0}, b_{0}\right) \neq(0,0)$ and $\left(a_{n}, b_{n}\right) \neq(0,0)$. A Möbius transformation close to the identity map can be written as $M(z)=(\alpha z+\beta) /(1+\gamma z)$ and then $M^{-1}(z)=(-z+\beta) /(\gamma z-\alpha)$. Then $M^{-1} \circ f \circ M$ is a locally (complex) three-dimensional manifold in the space of rational maps. To see this fix three special points $p_{1}, p_{2}, p_{3}$ of $f$ (say periodic points or critical points). Then $(\alpha, \beta, \gamma) \mapsto\left(M^{-1}\left(p_{1}\right), M^{-1}\left(p_{2}\right), M^{-1}\left(p_{3}\right)\right) \in \overline{\mathbb{C}}^{3}$ forms a complex three-dimensional manifold through $\left(p_{1}, p_{2}, p_{3}\right) \in \overline{\mathbb{C}}^{3}$ consisting of the corresponding special points for $M^{-1} \circ f \circ M$. 
5. In [Tsu], Masata Tsujii proved that if $Q_{t}(x)=t-x^{2}$ with $t$ real is a Collet-Eckman map (i.e. $\liminf _{n \rightarrow \infty}\left|D Q_{t}^{n}\left(Q_{t}(0)\right)\right|^{1 / n}>1$ ) then

$$
\lim _{n \rightarrow \infty} \frac{\left.\frac{\partial}{\partial s}\left(Q_{s}^{n}(0)\right)\right|_{s=t}}{D Q_{t}^{n-1}\left(Q_{t}(0)\right)}>0 .
$$

This implies our result in the real quadratic case. It seems that his method really requires the parameter $t$ to be real.

Using the results of Tan Lei [TL] one can deduce from the main theorem similarities of the Julia set and the parameter space.

2. Proof in the case where all critical points of $f$ are eventually mapped to repelling periodic points. Even though the general case is not much more difficult, we shall first prove this case, because in this case the proof is a little more concrete. That $c_{i}$ and $p_{i}$ depend analytically on $\lambda$ is simply a consequence of the implicit function theorem. So it remains to show that the assumption that the family is normalized implies that

$$
\lambda \mapsto\left(f^{k_{1}}\left(c_{1}(\lambda)\right)-p_{1}(\lambda), \ldots, f^{k_{n}}\left(c_{n}(\lambda)\right)-p_{n}(\lambda)\right)
$$

is a local immersion. The main ingredient for proving this is the following

TheOREM 2.1 (Uniqueness part of Thurston's realization theorem). Let $f, \tilde{f}: \overline{\mathbb{C}} \rightarrow \overline{\mathbb{C}}$ be critically finite rational maps which are combinatorially equivalent. Then $f$ and $\widetilde{f}$ are conjugate by some Möbius transformation.

Here $f$ and $\tilde{f}$ are called combinatorially equivalent if there exist two orientation preserving homeomorphisms $\theta_{1}, \theta_{2}: \overline{\mathbb{C}} \rightarrow \overline{\mathbb{C}}$ such that $f \circ \theta_{1}=$ $\theta_{2} \circ \widetilde{f}, \theta_{1}=\theta_{2}$ on $P(f), \theta_{i}(P(f))=P(\widetilde{f})$ and $\theta_{1}, \theta_{2}$ are isotopic relative to $P(f)$. (Assume that $P(f)$ and $P(\widetilde{f})$ are finite in this definition.)

We shall apply this uniqueness result, by using the following hyperbolic structure near periodic points.

Lemma 2.1. For each repelling periodic point $p$ of period $l$ there exist an open topological disc $U$, two open topological discs $U^{0}, U^{1}$ whose closures are disjoint and contained in $U$ and two positive integers $s_{1}, s_{2}$ such that $f^{s_{j}}: U^{j} \rightarrow U$ is a diffeomorphism for $j=1,2$. Here $p \in U^{0}$ and $s_{0}=l$. We can assume that $f^{s_{j}} \mid U_{j}$ are the first return maps of $f$ to $U$. Moreover, we can choose $U$ with arbitrarily small diameter.

Pr o of. Take a small open Euclidean disc $V$ around $p$ so that one branch of $f^{-l}$ maps $V$ compactly into $V$. Call this inverse branch $g$. Let $V_{-i}=g^{i}(V)$. So $p \in V_{-i}$ and $V_{-(i+1)} \subset V_{-i}$. Since the Julia set is perfect, and the preimages of non-exceptional points are dense in the Julia set, there exists an integer $i$ and some preimage $x$ of $p$ which is contained in $V_{-i} \backslash V_{-(i+1)}$. We can assume that $x$ is not a preimage of a critical point and that the first 
iterate of $x$ which enters $V_{-i}$ is $p$. Let $m$ be so that $f^{m}(x)=p$. Hence there exists a small disc $B$ around $x$ which is mapped by $f^{m}$ diffeomorphically onto some $V_{i^{\prime}}$ with $i^{\prime} \geq i$. We can arrange it so that $f^{t}(B) \cap V_{i^{\prime}}=\emptyset$ for $t=0,1, \ldots, m-1$. So define $U=V_{-i^{\prime}}, U^{0}=V_{-\left(i^{\prime}+1\right)}$ and $U^{1}=g^{i^{\prime}-i}(B)$. Then $U^{1} \subset V_{-i^{\prime}} \backslash V_{-\left(i^{\prime}+1\right)}=U \backslash U^{0}$ and $f^{l}: U^{0} \rightarrow U, f^{m+\left(i^{\prime}-i\right) l}: U^{1} \rightarrow U$ are both diffeomorphisms. By construction, each of these maps is a first return to $U$, and $U^{0}, U^{1}$ have disjoint closures.

$F \mid U^{j}:=f^{s_{j}}$ defines a polynomial-like $F: U^{0} \cup U^{1} \rightarrow U$. The set

$$
\Lambda=\left\{z \in U^{0} \cup U^{1}: F^{j}(z) \in U^{0} \cup U^{1}\right\}
$$

forms a hyperbolic Cantor set (by putting the Poincaré metric on $U$, the two maps $\left(F \mid U^{i}\right)^{-1}: U \rightarrow U^{i}$ become strictly contracting). The dynamics of $F: \Lambda \rightarrow \Lambda$ is conjugate to the shift $\sigma:\{0,1\}^{\mathbb{N}} \rightarrow\{0,1\}^{\mathbb{N}}$ where the conjugacy is $A(z)=\left(a_{m}(z)\right)_{m \geq 0}$ with $a_{m}(z)$ defined so that $f^{m}(z) \in U^{a_{m}(z)}$. The previous lemma implies that there exists a hyperbolic Cantor set $\Lambda$ containing $p$, and that $p$ is the limit of a sequence of periodic points $q_{i} \in \Lambda$. The next lemma shows that this structure persists for nearby maps.

Lemma 2.2. Let $f=f_{\lambda_{0}}$ be as before. Then there exists $K<\infty$ and a neighbourhood $W$ of $\lambda_{0}$ such that for each $\lambda \in W$,

- there are branches of $f_{\lambda}^{-s_{i}} \mid U$ which are $\varepsilon$-close to $\left(f \mid U_{i}\right)^{-s_{i}}$;

- if we define $U_{\lambda}^{i}:=\left(f_{\lambda}^{-s_{i}}(U)\right)$ then $F_{\lambda} \mid U_{\lambda}^{i}=f_{\lambda}^{s_{i}}$ still defines a polynomial-like map $F_{\lambda}: U_{\lambda}^{0} \cup U_{\lambda}^{1} \rightarrow U$;

- there exists a family of $K$-quasiconformal $h_{\lambda}: U \rightarrow U$ depending continuously on $\lambda$ and with $h_{0}=\mathrm{id}$ so that $h_{\lambda}$ sends $\Lambda$ onto $\Lambda_{\lambda}$ and $h_{\lambda}: \Lambda \rightarrow \Lambda_{\lambda}$ conjugates $F: \Lambda \rightarrow \Lambda$ to $F_{\lambda}: \Lambda_{\lambda} \rightarrow \Lambda_{\lambda}$;

- for each $z \in \Lambda$, the map $W \ni \lambda \mapsto h_{\lambda}(z) \in U$ is holomorphic.

Pro of. This can be proved easily by applying the $\lambda$-Lemma (see [MSS]). It is also easy to prove it by hand. Let $h_{\lambda}^{(0)}: U \rightarrow U$ be a family of diffeomorphisms depending holomorphically on $\lambda$ which map $U \rightarrow U, U^{0} \rightarrow U_{\lambda}^{0}$, $U^{1} \rightarrow U_{\lambda}^{1}$, and so that $h_{0}^{(0)}(0)=\mathrm{id}$ and

$$
h_{\lambda}^{(0)}(0) \circ f^{s_{i}}=f_{\lambda}^{s_{i}} \circ h_{\lambda}^{(0)}(0)
$$

on $\partial U^{i}, i=1,2$. Provided $\lambda$ is close to $\lambda_{0}$ this is possible, and moreover one can find $K$ such that $h_{\lambda}^{(0)}$ is $K$-quasiconformal. Next define $h_{\lambda}^{(1)}$ to be equal to $h_{\lambda}^{(0)}$ outside $U^{0} \cup U^{1}$ and to $f_{\lambda}^{-s_{i}} \circ h_{\lambda}^{(0)} \circ f^{s_{i}}$ on $U^{i}$. Because of $(2.2), h_{\lambda}^{(1)}$ is a continuous map on $U$. It is also $K$-quasiconformal. Continuing this pullback construction we get a sequence of $K$-quasiconformal homeomorphisms $h_{\lambda}^{(n)}$ : $U \rightarrow U$ such that

$$
h_{\lambda}^{(n)} \circ F=F_{\lambda} \circ h_{\lambda}^{(n)}
$$


outside $\Lambda(n)=\left\{z \in U: z, \ldots, F^{n}(z) \in U^{0} \cup U^{1}\right\}$. Note that $h_{\lambda}^{(n)}$ agrees with $h_{\lambda}^{(n-1)}$ outside $\Lambda(n-1)$. Hence as $n$ tends to infinity, $h_{\lambda}^{(n)}$ converges to a $K$ quasiconformal homeomorphism $h_{\lambda}$ conjugating $F: \Lambda \rightarrow \Lambda$ to $F: \Lambda_{\lambda} \rightarrow \Lambda_{\lambda}$. For each $z \in \Lambda, h_{\lambda}(z)$ is the limit of $\phi_{\lambda}^{\left(a_{n}\right)} \circ \phi_{\lambda}^{\left(a_{n-1}\right)} \circ \ldots \circ \phi^{\left(a_{0}\right)}(x)$ where $x$ is an arbitrary point of $U,\left(a_{m}\right)$ is the coding $A(z)$ and $\phi_{\lambda}^{(i)}:=\left(f_{\lambda}^{s_{i}} \mid U_{\lambda}^{i}\right)^{-1}$. Since all these maps are contractions depending holomorphically on $\lambda$, it follows that for each fixed $z \in \Lambda$, the map $W \ni \lambda \mapsto h_{\lambda}(z)$ is holomorphic.

Note that the conjugacy is so that $z \in \Lambda$ and $h_{\lambda}(z) \in \Lambda_{\lambda}$ have the same coding by the map $A$ above (where in the definition of the coding of $\Lambda_{\lambda}$ we have of course to take $a_{m}(\widetilde{z})$ so that $f_{\lambda}^{m}(\widetilde{z}) \in U_{\lambda}^{a_{m}(\widetilde{z})}$ for the coding of $\left.\Lambda_{\lambda}\right)$. In other words, when we fix for example a periodic $q \in \Lambda$ then there exists a unique corresponding periodic point $q_{\lambda}=h_{\lambda}(q) \in \Lambda_{\lambda}$ for each $\lambda$ sufficiently close to $\lambda_{0}$. The map $\lambda \mapsto q_{\lambda}$ is holomorphic.

Let now $f=f_{\lambda_{0}}$ be a critically finite rational map. Associate polynomiallike maps $F_{i}: U^{0}\left(p_{i}\right) \cup U^{1}\left(p_{i}\right) \rightarrow U\left(p_{i}\right)$ with each of the periodic points $p_{i}=f^{k_{i}}\left(c_{i}\right)$ such that $F_{i} \mid U^{j}\left(p_{i}\right)=f^{s_{i, j}}$ are first return maps of $f$ to $U\left(p_{i}\right)$. Let $\Lambda\left(p_{i}\right)$ be the corresponding Cantor sets and let $W$ be a neighbourhood of $\lambda_{0}$ such that the previous lemma holds for each of the periodic points $p_{i}$.

Proposition 2.1. There exists a neighbourhood $W$ of $\lambda_{0}$ with the following property. Assume that $\lambda, \lambda^{\prime} \in W$ and that $f_{\lambda}$ and $f_{\lambda^{\prime}}$ are so that

$$
f_{\lambda}^{k_{i}}\left(c_{i}(\lambda)\right) \in \Lambda_{\lambda}\left(p_{i}\right) \quad \text { and } \quad f_{\lambda^{\prime}}^{k_{i}}\left(c_{i}\left(\lambda^{\prime}\right)\right) \in \Lambda_{\lambda^{\prime}}\left(p_{i}\right)
$$

are both periodic points of the same period and in fact correspond to one and the same periodic point $q_{i} \in \Lambda\left(p_{i}\right)$ of $f=f_{\lambda_{0}}$, i.e., are respectively equal to $h_{\lambda}\left(q_{i}\right)$ and $h_{\lambda^{\prime}}\left(q_{i}\right)$. Then $f_{\lambda}$ and $f_{\lambda^{\prime}}$ are conjugate via a Möbius transformation.

Note that the period of the periodic orbit from the above proposition tends necessarily to infinity as $\lambda, \lambda^{\prime}$ tend to $\lambda_{0}$.

Proof (of Proposition 2.1). SteP 1. Take $U\left(p_{i}\right)$ so that they are all disjoint and take the family of homeomorphisms $h_{\lambda}: U\left(p_{i}\right) \rightarrow U\left(p_{i}\right)$ from Lemma 2.2. Next let $\theta=h_{\lambda^{\prime}} \circ h_{\lambda}^{-1}$. This homeomorphism conjugates the polynomial-like maps

$$
F_{i, \lambda}: U_{\lambda}^{0}\left(p_{i}\right) \cup U_{\lambda}^{1}\left(p_{i}\right) \rightarrow U\left(p_{i}\right)
$$

to

$$
F_{i, \lambda^{\prime}}: U_{\lambda^{\prime}}^{0}\left(p_{i}\right) \cup U_{\lambda^{\prime}}^{1}\left(p_{i}\right) \rightarrow U\left(p_{i}\right) .
$$

Note that $\theta \mid \partial U\left(p_{i}\right)=\mathrm{id}$ and $F_{\lambda^{\prime}} \circ \theta=\theta \circ F_{\lambda}$ on $\bigcup_{i, j} U_{\lambda}^{j}\left(p_{i}\right)$.

SteP 2. We have $F_{\lambda} \mid U_{\lambda}^{j}\left(p_{i}\right)=f_{\lambda}^{s_{i, j}}$. Let $W_{i, \lambda}$ be the union of the first $s_{i, 0}-1$ iterates of $U_{\lambda}^{0}\left(p_{i}\right)$ together with the first $s_{i, 1}-1$ iterates of $U_{\lambda}^{1}\left(p_{i}\right)$. 
(Note that we may assume that all these iterates are disjoint, since $F_{\lambda} \mid U_{\lambda}^{j}\left(p_{i}\right)$ is a first return map.) Define $W_{i, \lambda^{\prime}}$ similarly. Extend $\theta$ to a homeomorphism $\bigcup W_{i, \lambda} \rightarrow \bigcup W_{i, \lambda^{\prime}}$ so that $f_{\lambda^{\prime}} \circ \theta(z)=\theta \circ f_{\lambda}(z)$ whenever $z \in \bigcup W_{i, \lambda}$.

STEP 3. Take a disc $V_{i, j}$ of radius $r$ centred at $f^{j}\left(c_{i}\right)$ for $j=0, \ldots, k_{i}-1$. Provided $\lambda, \lambda^{\prime}$ are sufficiently close to $\lambda_{0}$, one has $f_{\lambda}^{j}\left(c_{i}(\lambda)\right), f_{\lambda^{\prime}}^{j}\left(c_{i}\left(\lambda^{\prime}\right)\right)$ $\in V_{i, j}$. Assume that $r>0$ is so small that all these sets $V_{i, j}$ are pairwise disjoint (or coincide) and are disjoint from $\bigcup W_{i, \lambda}$ and $\bigcup W_{i, \lambda^{\prime}}$. Now choose a $\widetilde{K}$-quasiconformal homeomorphism $\theta: V_{i, j} \rightarrow V_{i, j}$ which is equal to the identity on the boundary of $V_{i, j}$ and so that it maps $f_{\lambda}^{j}\left(c_{i}(\lambda)\right)$ onto $f_{\lambda^{\prime}}^{j}\left(c_{i}\left(\lambda^{\prime}\right)\right)$. Next define $\theta=$ id outside $\bigcup\left(V_{i, j} \cup W_{i}\right)$. Thus $\theta: \overline{\mathbb{C}} \rightarrow \overline{\mathbb{C}}$ becomes a $\widetilde{K}$-quasiconformal homeomorphism which sends the post-critical set of $f_{\lambda}$ to that of $f_{\lambda^{\prime}}$. Note that we can choose $\widetilde{K}$ close to one (i.e., $\theta$ close to a Möbius map) provided $\lambda, \lambda^{\prime}$ are close to $\lambda_{0}$.

STEP 4. Let us now assume that the neighbourhood $W$ is small and $\widetilde{K}$ is close to one. Then define a conjugacy $\phi$ between $f_{\lambda}$ and $f_{\lambda^{\prime}}$ as the limit of a sequence of quasiconformal homeomorphisms $\theta_{n}$. Here we define $\theta_{n}$ inductively as follows. Let $\theta_{0}=\theta$ and given a $\widetilde{K}$-quasiconformal homeomorphism $\theta_{n}$ which sends the post-critical set of $f_{\lambda}$ to that of $f_{\lambda^{\prime}}$, define a homeomorphism $\theta_{n+1}$ with the same properties as follows. Take $z$ and consider the set $f_{\lambda^{\prime}}^{-1} \circ \theta_{n} \circ f_{\lambda}(z)$. Provided $z \notin V_{i, 0}$, this set contains precisely one point $z^{\prime}$ which is close to $z$. (Provided $\varepsilon>0$ is sufficiently small, there exists a neighbourhood $W$ of $\lambda_{0}$ such that if $\lambda, \lambda^{\prime} \in W$, then $\theta_{n}$ is close to the identity map and so there is a unique point $z^{\prime} \in f_{\lambda^{\prime}}^{-1} \circ \theta_{n} \circ f_{\lambda}(z)$ which is $\varepsilon$-close to $z$. This does not hold when $z \in V_{i, 0}$ because $V_{i, 0}^{\prime}:=\theta\left(f_{\lambda}\left(V_{i, 0}\right)\right)$ contains a critical value of $f_{\lambda^{\prime}}$.) So define $\theta_{n+1}(z)=z^{\prime}$ in this way for $z \notin \bigcup V_{i, 0}$. Thus $\theta_{n+1}$ is a homeomorphism sending the complement of $\bigcup V_{i, 0}$ to the complement of $\bigcup V_{i, 0}^{\prime}$. Since $\theta_{n+1}$ is now already defined from $\partial V_{i, 0}$ to $\partial V_{i, 0}^{\prime}$, this map extends uniquely to a homeomorphism sending $V_{i, 0}$ to $V_{i, 0}^{\prime}$ with $\theta_{n+1}(z) \in f_{\lambda^{\prime}}^{-1} \circ \theta_{n} \circ f_{\lambda}(z)$ (since the map is prescribed on the boundary, continuity of $\theta_{n+1}$ forces uniqueness in the choice of the preimages). Note that $\theta_{n+1}$ is again a $\widetilde{K}$-quasiconformal homeomorphism sending the post-critical set of $f_{\lambda}$ to that of $f_{\lambda^{\prime}}$ and $\theta_{n+1} \circ f_{\lambda^{\prime}}=\theta_{n} \circ f_{\lambda}(z)$. Now taking a subsequence of $\theta_{n}$ which converges to some $\widetilde{K}$-quasiconformal homeomorphism $\phi$ we get $\phi \circ f_{\lambda^{\prime}}=\phi \circ f_{\lambda}(z)$. From Thurston's uniqueness result, it follows that $f_{\lambda^{\prime}}$ and $f_{\lambda}$ are conjugate by a Möbius transformation.

We shall use the following

Lemma 2.3. Let $G=\left(G_{1}, \ldots, G_{n}\right): V \rightarrow \mathbb{C}^{n}$ be a complex analytic map where $\lambda_{0} \in V \subset \mathbb{C}^{n}$. Assume that $G$ has an isolated zero at $\lambda_{0}$. Then there 
is (locally) a univalent parameterization

$$
\Psi: \mathbb{C} \rightarrow\left\{z: G_{2}(z)=\ldots=G_{n}(z)=0\right\}
$$

with $\Psi(0)=\lambda_{0}$.

Proof. This is a consequence of the Uniformization Theorem (see for example [AGLV]).

Proof of the Main Theorem in the critically finite case. Assume that $f_{\lambda}$ is a normalized family depending on $\lambda \in W \subset \mathbb{C}^{n}$, and consider the map $G: W \rightarrow \mathbb{C}^{n}$ defined by

$$
\lambda \mapsto\left(f_{\lambda}^{k_{1}}\left(c_{1}(\lambda)\right)-p_{1}(\lambda), \ldots, f_{\lambda}^{k_{n}}\left(c_{n}(\lambda)\right)-p_{n}(\lambda)\right) .
$$

Let us first show that $G$ has an isolated zero at $\lambda_{0}$. Indeed, otherwise there exist a sequence of parameters $\lambda(j)$ converging to $\lambda_{0}$ such that $G(\lambda(j))$ vanishes. This means that for each fixed $i$, each $f_{\lambda(j)}^{k_{i}}\left(c_{i}(\lambda(j))\right)$ corresponds to the same periodic point. Hence by Proposition 2.1 the maps $f_{\lambda(j)}$ all coincide up to a Möbius transformation, contradicting the assumption that $f_{\lambda}$ is a normalized family.

So we may assume that the zero of $G$ is isolated. Let $G_{1}, \ldots, G_{n}$ be the coordinate functions of $G$ and let $Z_{i}$ be the set of $\lambda \in W$ for which $G_{j}(\lambda)=0$ for all $j \neq i$. Let $\Psi_{i}: \mathbb{C} \rightarrow Z_{i}$ be the univalent parameterization of $Z_{i}$ with $\Psi_{i}(0)=\lambda_{0}$.

Claim. $\left(G_{i} \circ \Psi_{i}\right)^{\prime}(0) \neq 0$.

Proof. Assume the claim is false. Choose a periodic point $q_{i}(\lambda) \in \Lambda_{\lambda}$ distinct from $p_{i}(\lambda)$ such that $R_{i}(\lambda):=q_{i}(\lambda)-p_{i}(\lambda)$ is close to zero for all $\lambda \epsilon$ $W$. The existence of such periodic points follows from Lemmas 2.1 and 2.2. Note that $p_{i}(\lambda) \neq q_{i}(\lambda)$ for all $\lambda \in W$. Note that $G_{i}(\lambda)=f_{\lambda}^{k_{i}}\left(c_{i}(\lambda)\right)-p_{i}(\lambda)$ and write

$$
\widetilde{G}_{i}(\lambda):=f_{\lambda}^{k_{i}}\left(c_{i}(\lambda)\right)-q_{i}(\lambda)=\left(f_{\lambda}^{k_{i}}\left(c_{i}(\lambda)\right)-p_{i}(\lambda)\right)+\left(p_{i}(\lambda)-q_{i}(\lambda)\right)
$$

and

$$
U_{i}(\lambda):=\left(p_{i}(\lambda)-q_{i}(\lambda)\right)
$$

Then $\widetilde{G}_{i}=G_{i}+U_{i}$. Choose $\delta>0$ so small that $\Psi(t) \in W$ for all $t \in$ $B_{\delta}(0)$. Since we have assumed that the claim does not hold, $G_{i} \circ \Psi_{i}$ has a zero of multiplicity $d \geq 2$ at 0 . Hence $G_{i} \circ \Psi_{i}(t)=t^{d} g(t)$ where $g(t)$ is some holomorphic function on the $\operatorname{disc} B_{\delta}(0)$ with $g(0) \neq 0$. By choosing $\delta$ sufficiently small, we may assume that $g(t) \neq 0$ for all $t \in B_{\delta}(0)$. Let us show that $B_{\delta}(0) \ni t \mapsto \widetilde{G}_{i} \circ \Psi_{i}(t)$ has several distinct zeros provided we choose $q_{i}(\lambda)$ sufficiently close to $p_{i}(\lambda)$ (which means that $U_{i}$ is close to 0 ). Note that

$$
0=\widetilde{G}_{i} \circ \Psi_{i}(t)=G_{i} \circ \Psi_{i}(t)+U_{i} \circ \Psi_{i}(t)=t^{d} g(t)+U_{i} \circ \Psi_{i}(t)
$$


is equivalent to

$$
t^{d} \frac{g(t)}{u(t)}=-1
$$

where $u(t)=U_{i} \circ \Psi_{i}(t)$ (note that $u(t) \neq 0$ for $t \in B_{\delta}(0)$ ). Since $g(t) / u(t) \neq 0$ for all $t \in B_{\delta}(0)$, it follows that the closed curve $\partial B_{\delta / 2}(0) \ni t \mapsto \varrho(t):=$ $g(t) / u(t)$ has winding number zero, and that $\partial B_{\delta / 2}(0) \ni t \mapsto t^{d} \varrho(t)$ has winding number $d$. Moreover, the image of $\partial B_{\delta / 2}(0) \ni t \mapsto t^{d} \varrho(t)$ is outside the ball $B_{3}(0)$ (provided we choose $q_{i}(\lambda)$ close enough to $p_{i}(\lambda)$ because then $u(t)$ is small but not zero in $\left.B_{\delta}(0)\right)$. It follows that there are $d$ points $t \in B_{\delta / 2}(0)$ such that $t^{d} \varrho(t)=-1$. To show that these points do not occur with higher multiplicity we need the following

SubCLAIm. -1 is not a critical value of the map $B_{\delta / 2}(0) \ni t \mapsto t^{d} \varrho(t)=$ -1 provided we choose $\delta>0$ sufficiently small and $q_{i}$ sufficiently close to $p_{i}$ (which means that $u(t)$ is close to zero).

Proof. The homeomorphism $h_{t}$ which conjugates $f_{\Psi_{i}(0)}$ to $f_{\Psi_{i}(t)}$ is $K$ quasiconformal and maps $p_{i}\left(\Psi_{i}(0)\right), q_{i}\left(\Psi_{i}(0)\right)$ to $p_{i}\left(\Psi_{i}(t)\right), q_{i}\left(\Psi_{i}(t)\right)$. By normalizing we can assume that $\widetilde{h}_{t}$ maps $u(0)$ to $u(t)$ and fixes $0,1, \infty$. Therefore, according to Lemma 4.1 in the appendix, there exists $K^{\prime}$ so that for all $t \in B_{\delta}(0)$,

$$
\frac{1}{K^{\prime}}|u(0)|^{1 / K} \leq|u(t)| \leq K^{\prime}|u(0)|^{1 / K}
$$

and (going along a line through 0 to $u(0) /|u(0)|$ ) this implies that for $s \in(0,1)$,

$$
\left|\arg \left(\widetilde{h}_{t}\left(s \frac{u(0)}{|u(0)|}\right)\right)-\arg \left(\widetilde{h}_{t}\left(\frac{u(0)}{|u(0)|}\right)\right)\right| \leq K^{\prime} \cdot \frac{1}{-\log s} .
$$

Write $u(t)=\exp (v(t))$ (since $u(t)$ is close to zero, $\Re(v(t))$ is close to $-\infty$ ). Choose $v(t)$ so that $\Im(v(0)) \in[0,2 \pi)$. The previous inequalities show that

$$
\begin{gathered}
K \Re(v(0)) \leq \Re(v(t)) \leq \frac{1}{K} \Re(v(0)), \\
|\Im(v(t))| \leq K^{\prime}|\Re(v(0))|+K^{\prime \prime} \leq K^{\prime \prime \prime}|\Re(v(0))|,
\end{gathered}
$$

provided $u(0)$ is close enough to 0 (which means that $\Re(v(0)$ ) is close to $-\infty$ ). In particular,

$$
|v(t)| \leq K_{1}|\Re(v(0))|
$$

for $t \in B_{\delta}(0)$. Because of the Cauchy integral formula this implies

$$
\left|v^{\prime}(t)\right| \leq K_{2}|\Re(v(0))|
$$

for $|t| \leq \delta / 2$. So let us assume by contradiction that $t^{d} \varrho(t)=-1$ and that $t$ 
is a critical point of $t \mapsto t^{d} \varrho(t)$. Since $\varrho(t)=g(t) / u(t)$ this gives

$$
0=d t^{d-1} \varrho(t)+t^{d} \frac{g^{\prime}(t) u(t)-g(t) u^{\prime}(t)}{[u(t)]^{2}},
$$

i.e.,

$$
0=d g(t)+t g^{\prime}(t)-t g(t) \frac{u^{\prime}(t)}{u(t)}=d g(t)+t g^{\prime}(t)-t g(t) v^{\prime}(t) .
$$

Since $g$ is non-zero on $B_{\delta}(0)$, this means that $\left|t v^{\prime}(t)\right| \geq K_{3}>0$ for $t \in B_{\delta}(0)$ provided $\delta>0$ is sufficiently small (where $K_{3}$ only depends on $g$ and not on which point $q_{i}$ we choose). From (2.5) this gives

$$
|t| \cdot|\Re(v(0))| \geq K_{4}>0
$$

and, since $-1=t^{3} \varrho(t)$,

$$
1=|t|^{d}|\varrho(t)| \geq|t|^{d} \exp \left(-K_{5} \Re(v(0))\right)
$$

(where we used (2.4)). Clearly these two inequalities cannot both hold when $|u(0)|$ is small (because then $\Re(v(0))$ is close to $-\infty$ ). This completes the proof of the subclaim.

From all this it follows that $t^{d} g(t) / r(t)=-1$ has $d$ distinct solutions inside the disc $B_{\delta / 2}(0)$. In particular, if $d>1$ then $\widetilde{G}_{i} \circ \Psi_{i}$ has at least two distinct zeros $t, t^{\prime} \in B_{\delta / 2}(0)$. It follows that $\lambda=\Psi_{i}(t)$ and $\lambda^{\prime}=\Psi_{i}\left(t^{\prime}\right)$ are distinct zeros of $\widetilde{G}_{i}$. Using Proposition 2.1 we again obtain a contradiction if $d>1$. This concludes the proof of the claim.

From the claim we see that $\Psi_{i}^{\prime}(0) \neq 0$ and $Z_{i}$ is a smooth curve in $\mathbb{C}^{n}$ through $\lambda_{0} \in \mathbb{C}^{n}$. Hence we also deduce that $D G_{i}\left(v_{i}\right) \neq 0$ where $v_{i}$ is any unit tangent vector in $T_{\lambda_{0}} Z_{i} \subset T_{\lambda_{0}} \mathbb{C}^{n}$. Since $G$ maps $Z_{i}$ to the $i$ th coordinate plane in $\mathbb{C}^{n}$ it follows that the tangent lines at $\lambda_{0}$ of the analytic curves $Z_{i}$ are linearly independent, i.e. $v_{1}, \ldots, v_{n}$ forms a basis of $T_{\lambda_{0}} \mathbb{C}^{n}$. It follows that $D G^{\prime}\left(\lambda_{0}\right) \neq 0$ and that $G$ is a local diffeomorphism near $\lambda_{0}$.

3. Proof in the Misiurewicz case. The main tools in the Misiurewicz case are the following two theorems of Mañé (for the proofs, see [Ma2] and also $[\mathrm{ST}])$.

THeOREM 3.1. Let $f: \overline{\mathbb{C}} \rightarrow \overline{\mathbb{C}}$ be a rational map. If a point $x \in J(f)$ is not a parabolic periodic point and is not contained in the $\omega$-limit set of a recurrent critical point, then for all $\varepsilon>0$ there exists a neighbourhood $U$ of $x$ such that

(1) for every $k \geq 0$, each connected component of $f^{-k}(U)$ has spherical diameter $\leq \varepsilon$ 
(2) there exists $N>0$ depending only on $f$ such that for all $k \geq 0$ and every connected component $V^{\prime}$ of $f^{-k}(U)$,

$$
\operatorname{deg}\left(f^{k}: V^{\prime} \rightarrow U\right) \leq N
$$

(3) for all $\varepsilon_{1}>0$ there exists $k_{0}$ such that every connected component of $f^{-k}(U)$ for $n \geq k_{0}$ has spherical diameter $\leq \varepsilon_{1}$.

Theorem 3.2. Let $f: \overline{\mathbb{C}} \rightarrow \overline{\mathbb{C}}$ be a rational map and $\Lambda \subset J(f)$ a compact invariant set not containing critical points and parabolic periodic points. Then $\Lambda$ is either an expanding hyperbolic set or $\Lambda \cap \omega(c) \neq \emptyset$ for some recurrent critical point $c$ of $f$.

In order to prove the main theorem we shall generalize all the steps from the previous section. First note that a Misiurewicz map cannot have a Siegel disc or a Herman ring $F$. Indeed, since no critical point of a Misiurewicz map is recurrent, we can apply Theorem 3.1 to any point $x$ in $\partial F$. But property (3) from that theorem clearly cannot hold for a neighbourhood $U$ of $x$ since $U$ would intersect the Siegel disc or Herman ring. So a Misiurewicz map $f$ cannot have non-hyperbolic periodic points (i.e., all eigenvalues have norm $\neq 1$ ). Let us also show that $f$ can have no periodic points with eigenvalues $|\mu|<1$. Indeed, a periodic attractor has a critical point in its immediate basin. Since $f$ is a Misiurewicz map, this critical point is eventually mapped into a periodic orbit. This is only possible if the critical point actually lies on the attracting periodic orbit. It follows that for a Misiurewicz map the only Fatou components correspond to basins of super-attracting periodic points.

TheOREM 3.3 (Uniqueness part of Thurston's realization theorem in the Misiurewicz case). Let $f, \widetilde{f}: \overline{\mathbb{C}} \rightarrow \overline{\mathbb{C}}$ be Misiurewicz maps which are $K$-quasiconformally conjugate. Moreover, assume that $f, \widetilde{f}$ are not Lattès maps. Then $f$ and $\widetilde{f}$ are conjugate by some Möbius transformation.

Proof. Let $h$ be the quasiconformal conjugacy between $f$ and $\widetilde{f}$. We may assume that the restriction of $h$ to the Fatou set is conformal. Indeed, let $c_{1}, \ldots, c_{s}$ be the critical points of $f$ which are not in the Julia set and let $\widetilde{c}_{i}=h\left(c_{i}\right)$. Since the maps are Misiurewicz, these critical points are either periodic or eventually mapped to a periodic critical point. Hence there exists a conformal conjugacy $h_{1}$ of $f$ from a neighbourhood $O$ of the $f$ forward orbits of $\left\{c_{1}, \ldots, c_{s}\right\}$ to a neighbourhood $\widetilde{O}$ of the $\widetilde{f}$-forward orbits of $\left\{\widetilde{c}_{1}, \ldots, \widetilde{c}_{s}\right\}$ with $\widetilde{h}\left(c_{i}\right)=\widetilde{c}_{i}$. (Here we use the fact that the map near a superattracting periodic orbit is locally conformally conjugate to a map of the form $z \mapsto z^{l}$ and moreover that critical points of $f$ have the same order as the corresponding critical points of $\widetilde{f}$ since these maps are conjugate.) Let $H$ be a $K^{\prime}$-quasiconformal homeomorphism which is equal to $h$ on a neighbourhood 
of $J(f)$ and coincides with $h_{1}$ near the $f$-forward orbits of $\left\{c_{1}, \ldots, c_{s}\right\}$. Next we can define a sequence of quasiconformal homeomorphisms $H_{n}$ such that $H_{n+1} \circ \widetilde{f}=f \circ H_{n}$. Since $H_{n}=H_{n+1}$ outside the basins of the critical points $c_{i}$ (and also on $\left.f^{-n}(O)\right), H_{n}$ converges to a conjugacy $H_{\infty}$ which is also $K^{\prime}$-quasiconformal. By construction $H_{\infty}$ is conformal on the basin of super-critical periodic points, and therefore outside the Julia set.

It is very easy to see that for a Misiurewicz map, $J(f)$ either is equal to $\overline{\mathbb{C}}$ or has zero Lebesgue measure. Indeed, assume that the Lebesgue measure of $J(f)$ is positive. Take a Lebesgue density point $y$ of $J(f)$ and a limit point $x$ of $f^{n_{i}}(y)$. Let $|A|$ denote the Lebesgue measure of a measurable set $A$. Fix two discs $U^{\prime} \subset U$ centred at $x$ where $U$ is taken so small that Theorem 3.1 holds. Using Mañé's Theorem 3.1 and applying Koebe at most $N$ times, we find that the component $B_{i}$ of $f^{-n_{i}}\left(U^{\prime}\right)$ which contains $y$ is "approximately round", and so this gives $\left|B_{i} \cap J(f)\right| /\left|B_{i}\right| \rightarrow 1$ and

$$
\left|U^{\prime} \cap J(f)\right| /\left|U^{\prime}\right|=\left|f^{n_{i}}\left(B_{i}\right) \cap J(f)\right| /\left|f^{n_{i}}\left(B_{i}\right)\right| \rightarrow 1 .
$$

Hence if $J(f)$ has positive Lebesgue measure then $J(f)$ contains an open set, and so $J(f)=\overline{\mathbb{C}}$. So if $J(f)$ is not equal to $\overline{\mathbb{C}}$ then $H_{\infty}$ is almost everywhere conformal and therefore a Möbius transformation.

So assume $J(f)=\overline{\mathbb{C}}$. If the quasiconformal distortion of $H_{\infty}$ does not vanish almost everywhere then it induces a measurable line field $\mu$ on the Julia set of $f$ which is invariant under $f$. Now we argue as in $[\mathrm{McM}]$ (see also [LS2]): take a density point $y$ of the support of $\mu$ at which $\mu$ is almost continuous (i.e., for any $\varrho>0$ and any sequence of balls $B_{i}$ centred at $y$, the set where the angle of the line field $\mu \mid B_{i}$ differs by more than $\varrho$ from some constant line field has Lebesgue measure $\theta_{i}\left|B_{i}\right|$ where $\theta_{i}$ tends to zero). Using again Mañé's Theorem 3.1 and Koebe in the same way as above, we obtain a small neighbourhood $U^{\prime}$ of $x$ on which $\mu$ defines a smooth foliation with at most $N$ singularities (because by Mañé's Theorem one can pass through the critical point at most $N$ times). From Lemma 3.16 of $[\mathrm{McM}]$ it follows that $f$ is a Lattès map, a contradiction.

We want to generalize Lemma 2.1 by showing that each critical value is a non-isolated point in some hyperbolic Cantor set (assuming that $f$ is a Misiurewicz map).

Lemma 3.1 (Analogue of Lemma 2.1). Assume that $f$ is a Misiurewicz map. Then there exists a neighbourhood $O$ of the set of critical points such that

$$
X_{O}=\left\{z: f^{n}(z) \notin O \text { for all } n \geq 0\right\}
$$

is a compact hyperbolic set and for each critical point $c$ there exists a sequence of preperiodic points in $X_{O}$ converging to $f(c)$. 
Proof. Since $f$ is Misiurewicz, any $x \in \omega(c)$ satisfies the assumptions of Theorems 3.1 and 3.2 ( $f$ has no neutral periodic points). Theorem 3.2 gives that $X_{O}$ is hyperbolic for any neighbourhood $O$ of $C(f)$. So let us prove the last assertion of this lemma. Since $f$ is a Misiurewicz map, there exists $\varepsilon>0$ such that the distance of all forward iterates of critical points to $C(f)$ is at least $2 \varepsilon$. If $c$ is eventually mapped to a repelling periodic orbit then Lemma 2.1 implies that one can choose $O$ so that $f(c)$ is a non-isolated point in $X_{O}$. So assume this is not the case and take $x \in \omega(c) \subset J(f)$. Let $U$ be the neighbourhood of $x$ from Theorem 3.1 corresponding to the above choice of $\varepsilon$. Let $y$ be a repelling periodic point in $U \cap J(f)$ and let $\varepsilon_{1} \in(0, \varepsilon)$ be so that the orbit of $y$ has distance $\geq \varepsilon_{1}$ to $C(f)$. Let $O$ be the $\varepsilon_{1}$-neighbourhood of $C(f)$. Since $x \in \omega(c)$, there exist arbitrarily large $\widehat{k}$ with $f^{\widehat{k}}(c) \in U$, and the preimages of $U$ have diameter $\leq \varepsilon$. In particular, there exists a point $y^{\prime}=y^{\prime}(\widehat{k})$ in the component of $f^{-\widehat{k}}(U)$ containing $c$ such that $f^{\widehat{k}}\left(y^{\prime}\right)=y$ (i.e., $y^{\prime}$ is preperiodic) and $\operatorname{dist}\left(f^{i}\left(y^{\prime}\right), f^{i}(f(c))\right) \leq \varepsilon$ for $i=0,1, \ldots, \widehat{k}$. In particular, this implies that $f^{i}\left(y^{\prime}\right)$ has distance $\geq \varepsilon^{\prime}$ to $C(f)$ for all $i \geq 1$ (by the definition of $\varepsilon$ and $\left.\varepsilon^{\prime}\right)$. From part (3) of Theorem 3.1, $y^{\prime}(\widehat{k})$ tends to $c$ as $\widehat{k} \rightarrow \infty$. The assertion of the lemma follows.

The persistence of $X_{O}$ under (small) changes of the parameter follows from the hyperbolicity of $X_{O}$ :

Lemma 3.2 (Analogue of Lemma 2.2 and Proposition 2.1). Let $f=f_{\lambda_{0}}$ be as before and let $X$ be a hyperbolic set. Then there exists a neighbourhood $W$ of $\lambda_{0}$ such that for $\lambda \in W$,

- there exists a family of $K$-quasiconformal $h_{\lambda}: \overline{\mathbb{C}} \rightarrow \overline{\mathbb{C}}$ depending continuously on $\lambda$ and with $h_{0}=$ id so that $X_{\lambda}=h_{\lambda}(X)$ and the restriction $h_{\lambda}: X \rightarrow X_{\lambda}$ conjugates $F: X \rightarrow X$ to $F_{\lambda}: X_{\lambda} \rightarrow X_{\lambda}$;

- for each $z \in X$, the map $W \ni \lambda \mapsto h_{\lambda}(z) \in U$ is holomorphic.

P r o of. Use Theorem III.1.6 of [MS] and the $\lambda$-Lemma (cf. also [McM]).

Proof of Main Theorem. Consider the map

$$
G(\lambda)=\left(f_{\lambda}\left(c_{1}(\lambda)\right)-\phi_{\lambda}\left(f\left(c_{1}\right)\right), \ldots, f_{\lambda}\left(c_{n}(\lambda)\right)-\phi_{\lambda}\left(f\left(c_{n}\right)\right)\right)
$$

and let $G_{i}$ be its coordinate functions. Then $G$ has an isolated zero at $\lambda_{0}$ because otherwise we get a sequence of Misiurewicz maps $f_{\lambda(n)}$ with $\lambda(n) \rightarrow \lambda_{0}$ and $G(\lambda(n))=0$. Because of Lemma 3.2 all these maps $f_{\lambda(n)}$ are quasiconformally conjugate to $G_{\lambda_{0}}$ and by Theorem 3.3 they are conformally conjugate. This contradicts the assumption that $f_{\lambda}$ is a normalized family.

So assume that $G$ has an isolated zero, and let $Z_{i}$ be the set of $\lambda \in W$ with $G_{j}(\lambda)=0$ for all $j \neq i$. As before, let $\Psi_{i}: \mathbb{C} \rightarrow Z_{i}$ be univalent parameterizations of $Z_{i}$ with $\Psi_{i}(0)=\lambda_{0}$. We again need to show that $\left(G_{i} \circ \Psi_{i}\right)^{\prime}(0) \neq 0$. Let us consider a few cases. 
CASE 1: The critical point $c_{i}$ is in the Julia set. In this case, by Lemma 3.1, there is a hyperbolic set containing hyperbolic periodic points $q_{i}$ arbitrarily close to $f\left(c_{i}\right)$. Thus the proof is identical to the proof in the previous section.

Case 2: The critical point $c_{i}$ is periodic (i.e., a super-attracting periodic point) of, say, period $l=l_{i}$. In this case, let $p_{i}(\lambda)$ be the periodic point of $f_{\lambda}$ with $p_{i}\left(\lambda_{0}\right)=c_{i}$. Note that $p_{i}(\lambda)$ and $c_{i}(\lambda)$ depend analytically on $\lambda$ by the implicit function theorem. Note that by the chain rule

$$
\left(f_{\lambda}^{l}\right)^{\prime}\left(p_{i}(\lambda)\right)=g(\lambda)\left(p_{i}(\lambda)-c_{i}(\lambda)\right)
$$

because the critical point of $f$ is non-degenerate. Here $g(\lambda)$ is non-zero. Now if

$$
t \mapsto \Psi_{i} \circ G_{i}(t)=\left(p_{i}\left(G_{i}(t)\right)-c_{i}\left(G_{i}(t)\right)\right)
$$

has derivative zero at $t=0$ then there are at least two parameters $\lambda=G_{i}(t)$ and $\lambda^{\prime}=G_{i}\left(t^{\prime}\right)$ arbitrarily close to $\lambda_{0}$ such that

$$
\left(f_{\lambda}^{l}\right)^{\prime}\left(p_{i}(\lambda)\right)=\left(f_{\lambda^{\prime}}^{l}\right)^{\prime}\left(p_{i}\left(\lambda^{\prime}\right)\right)
$$

(and are close to 0, i.e., the points are attracting). But then we conformally conjugate the restriction of $f_{\lambda}$ to a neighbourhood of $p_{i}(\lambda)$ to the restriction of $f_{\lambda^{\prime}}$ to a neighbourhood of $p_{i}\left(\lambda^{\prime}\right)$ in such a way that these neighbourhoods contain $f_{\lambda}\left(c_{i}(\lambda)\right)$ respectively $f_{\lambda^{\prime}}\left(c_{i}\left(\lambda^{\prime}\right)\right)$ and the conjugacy maps one critical point to the other. Then one can extend the conjugacy so that it becomes conformal on the basin of these attracting fixed points. But since the forward orbits of other critical points still remain outside neighbourhoods of the critical points (or are still periodic), we can then extend this to a quasiconformal conjugacy between $f_{\lambda}$ and $f_{\lambda^{\prime}}$; now by using the same proof as in Theorem 3.3 this shows that in fact $f_{\lambda}$ and $f_{\lambda^{\prime}}$ are conjugate via a Möbius transformation.

4. Appendix. In the next lemma we prove that a quasiconformal homeomorphism fixing 0 sends a line 0 to a curve through 0 which spirals no faster than logarithmically.

Lemma 4.1. For each $K \geq 0$ there exists $K^{\prime}<\infty$ with the following property. Let $h: \overline{\mathbb{C}} \rightarrow \overline{\mathbb{C}}$ be a $K$-quasiconformal homeomorphism fixing 0 , 1 and $\infty$. Then for $z \in \mathbb{C}, s>0, \phi \in \mathbb{R}$,

$$
\begin{gathered}
\frac{1}{K^{\prime}}|z|^{K} \leq|h(z)| \leq K^{\prime}|z|^{1 / K} \\
\left|\arg \left(h\left(s e^{i \phi}\right)\right)-\arg \left(h\left(e^{i \phi}\right)\right)\right| \leq K^{\prime} \cdot \frac{1}{-\log |s|} .
\end{gathered}
$$

(The difference of the arguments is well defined if we take a continuous lift of $(0,1) \ni s \mapsto \arg \left(h\left(s e^{i \phi}\right)\right)$.) 
If, for $r>0$ and $\phi \in \mathbb{R}$, we write $h\left(r e^{\phi i}\right)=h_{1}(r, \phi) e^{h_{2}(r, \phi) i}$ with $h_{1}(r, \phi) \geq 0$ and $h_{2}(r, \phi) \in \mathbb{R}$, then (4.7) gives

$$
\left|h_{2}(r, \phi)-h_{2}(1, \phi)\right| \leq \frac{K^{\prime}}{-\log r} .
$$

Proof. Let $\mathcal{F}$ be the family of $K$-quasiconformal homeomorphisms fixing $0,1, \infty$. By Theorem 2.1 of $[\mathrm{Le}], \mathcal{F}$ is an equicontinuous (and normal) family. From this it follows (see Theorem 2.4 of [Le]) that there exists $c(K)>0$ such that for any $r>0$,

$$
\frac{\max _{\phi}\left|h\left(r e^{i \phi}\right)\right|}{\min _{\phi}\left|h\left(r e^{i \phi}\right)\right|} \leq c(K) \text {. }
$$

According to Theorem II.3.1 of [LV], there exists a Hölder function $\phi_{K}: \mathbb{R}^{+} \rightarrow \mathbb{R}^{+}$such that for any $K$-quasiconformal homeomorphism $\widetilde{h}$ fixing 0 and sending the unit disc into itself, one has

$$
|\widetilde{h}(z)| \leq \phi_{K}(|z|) \text {. }
$$

Combining (4.9) and (4.10) gives (4.6). This also shows that the $h$-image of the annulus $\{1 / 2 \leq|z| \leq 1\}$ is contained in an annulus of the form $\{1 / M \leq|z| \leq M\}$ where $M$ only depends on $K$. Now consider the $h$-image of a line of the form $s e^{i \phi}$ with $s \in[1 / 2,1]$. By the normality of $\mathcal{F}$ the maximal variation of $[1 / 2,1] \ni s \mapsto \arg \left(h\left(s e^{i \phi}\right)\right)$ is bounded by some number $M^{\prime}$ which only depends on $K$ (otherwise there would be a sequence of maps $h_{n} \in \mathcal{F}$ for which the corresponding $M_{n}^{\prime}$ is at least $n$; such a sequence could not have a subsequence converging to a homeomorphism mapping $\{1 / 2 \leq|z| \leq 1\}$ into $\{1 / M \leq|z| \leq M\}$, contradicting the normality of $\mathcal{F})$. Now consider the $h$-image of an annulus $\left\{1 / 2^{k+1} \leq|z| \leq 1 / 2^{k}\right\}$. By rescaling $h$ as follows: $\widetilde{h}(w)=h\left(w / 2^{k}\right) / h\left(1 / 2^{k}\right)$, we get $\widetilde{h} \in \mathcal{F}$. Hence we can apply the previous argument to $\widetilde{h}$ and this shows that the variation of $\left[1 / 2^{k+1}, 1 / 2^{k}\right] \ni$ $s \mapsto \arg \left(h\left(s e^{i \phi}\right)\right)$ is again bounded by $M^{\prime}$. This means that the variation of $\left[1 / 2^{k}, 1\right] \ni s \mapsto \arg \left(h\left(s e^{i \phi}\right)\right)$ is bounded by $M^{\prime} k$, which proves the second inequality in the lemma.

\section{References}

[AGLV] V. I. Arnol'd, V. V. Goryunov, O. V. Lyashko and V. A. Vasil'ev, Singularity Theory I, Springer, 1998.

[DH] A. Douady and J. H. Hubbard, On the dynamics of polynomial-like mappings, Ann. Sci. Ecole Norm. Sup. 18 (1985), 287-343.

[Le] O. Lehto, Univalent Functions and Teichmüller Spaces, Grad. Texts in Math. 109, Springer, 1987.

[LV] O. Lehto and K. I. Virtanen, Quasiconformal Mappings in the Plane, Springer, 1973. 
[LS1] G. Levin and S. van Strien, Local connectivity of the Julia set of real polynomials, Ann. of Math. 147 (1998), 471-541.

[LS2] - - - Total disconnectedness of the Julia set of real polynomials, Astérisque, to appear.

[Ma1] R. Mañé, Hyperbolicity, sinks and measure in one dimensional dynamics, Comm. Math. Phys. 100 (1985), 495-524.

[Ma2] -, On a theorem of Fatou, Bol. Soc. Brasil. Mat. 24 (1993), 1-11.

[MSS] R. Mañé, P. Sad and D. Sullivan, On the dynamics of rational maps, Ann. Sci. Ecole Norm. Sup. 16 (1983), 193-217.

[McM] C. McMullen, Complex Dynamics and Renormalization, Ann. of Math. Stud. 135, Princeton Univ. Press, 1994.

[MS] W. de Melo and S. van Strien, One-Dimensional Dynamics, Ergeb. Math. Grenzgeb. 25, Springer, 1993.

[ST] M. Shishikura and L. Tan, Mañé's theorem, to appear.

[TL] L. Tan, Similarity between the Mandelbrot set and Julia sets, Comm. Math. Phys. 134 (1990), 587-617.

[Tsu] M. Tsujii, A simple proof for monotonicity of entropy in the quadratic family, Ergodic Theory Dynam. Systems, to appear.

Department of Mathematics

University of Warwick

Coventry CV4 7AL

United Kingdom

E-mail: strien@maths.warwick.ac.uk

Received 21 April 1999;

in revised form 23 September 1999 